

\title{
Separation of common and differential mode conducted emission: Power combiner/splitters
}

\author{
Dennis Nielsen \\ Technical University of Denmark \\ Department of Electrical Engineering \\ Ørsteds Plads, Building 349, room 008 \\ 2800 Kgs. Lyngby, Denmark \\ Email: deni@elektro.dtu.dk
}

\author{
Ole Cornelius Thomsen \\ Technical University of Denmark \\ Department of Electrical Engineering \\ Ørsteds Plads, Building 349, room 024 \\ 2800 Kgs. Lyngby, Denmark \\ Email: oct@elektro.dtu.dk
}

\author{
Michael A. E. Andersen \\ Technical University of Denmark \\ Department of Electrical Engineering \\ Ørsteds Plads, Building 349, room 034 \\ 2800 Kgs. Lyngby, Denmark \\ Email: ma@elektro.dtu.dk
}

\begin{abstract}
A conducted emission measurement contains a common and a differential mode component. Accurate separation of these two components is critical, when designing the input filter of a switch mode power supply. Many techniques exists for performing such separation. Some authors suggested the use of wideband transformers, while other prefer current probes. In this paper the use of commercial power splitters/combiners as noise separators are considered. The performance of the noise separators are analyzed and validated based on scattering parameters (S-parameters). Impedance and rejection ratios (common and differential mode) are shown. The results based on S-parameters are used to propose a complete noise separator design. This separator is verified through experimental measurements. Finally an example on how to use the separator, when measuring conducted noise is given.
\end{abstract}

Key words: EMI, Conducted emission, Power combiners/splitters, Differential and Common mode.

\section{INTRODUCTION}

Separation of conducted emission is a subject which has intrigue authors within the area of power electronics for many years [1], [2], [3], [4], [5], [6], [7], [8], [9], [10], [11]. Conducted emission can be divided into the categories of CM (Common Mode) and DM (Differential Mode). Each of these modes will in general refer to different parts of the EMI (Electromagnetic Interference) or input filter. In order to optimizes the design of such filters, correct diagnosis of CM and DM is key.

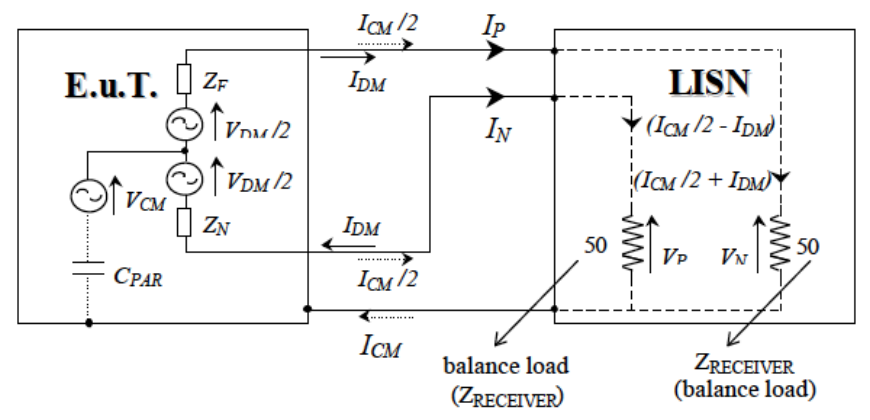

Figure 1: Measuring conducted emission [4].
The literature reports of many different techniques for separating conducted emission. Wide-band transformer are found in [9], [2], [3], [4], [12], while a combination of purely resistive networks and transformers are used in [6], [7], [8]. Current probes is another alternative as suggested in [1] and [11], while a software approach is introduced in [10]. A final option is active separation using operational amplifiers. Such configurations does however place significant demands on the bandwidth of the operational amplifiers.

In this paper power splitters/combiners will be used as noise separators. This idea was original proposed in [5] with an reported CMRR of around $-50 d B$ and DMRR of -50 $d B$ in the frequency region of $10 \mathrm{kHz}$ to $30 \mathrm{kHz}$. However the verification process used in [5] has been questioned by [2], as power combiners essential where used to validate power combiners. Further more the issue of maintaining the impedance requirements of the Line Impedance Stabilizing Network (LISN) is not addressed in [5]. The purpose of the LISN is to isolate the measurement from unwanted interference of the supply mains, and to define a impedance across the measuring point. This paper will cover the issue of maintaining the LISN impedance requirements, and validate the proposed noise separator using appropriate techniques.

\section{A. LISN}

Conducted emission on the mains port are measured using a LISN. CISPR 16-1-2 defines the most common type of LISN. Figure 2 shows a schematic of the network, while the impedance requirements are illustrated in figure 3 with the tolerances of $\pm 20 \%$ imposed [9]. Later in this paper an existing LISN will be modified in order to create the complete noise separator. The impedance of this LISN is also shown in figure 3 (before any modinification). Clearly, the requirements are fulfilled.

When adding a noise separator to the LISN, one should be extreme careful not to violate the impedance requirements of the network. The LISN is expected to see at pure resistive load of $50 \Omega$, corresponding to the load resistance of a 


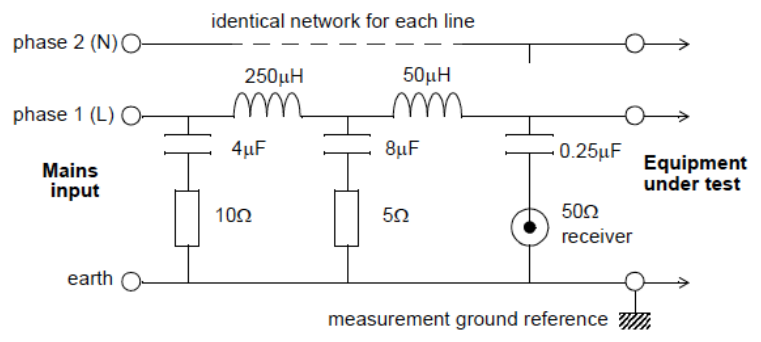

Figure 2: LISN [9].

spectrum analyzer. As shown in figure 3 , this will cause the $\mathrm{CM}$ emission to se $\frac{50 \Omega}{2}=25 \Omega$, while the DM emission sees $50 \Omega+50 \Omega=100 \Omega$.

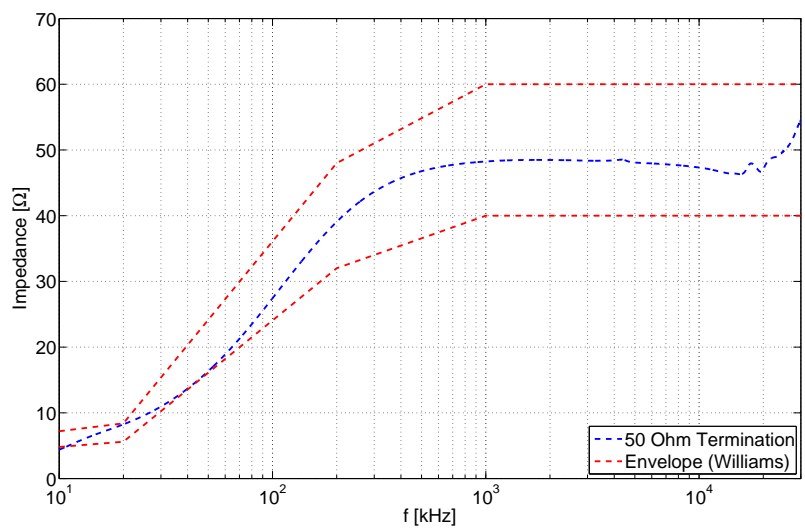

Figure 3: Input impedance of LISN.

\section{B. Power combiners/splitters}

A power combiner can be considered as a three port device, which adds the signals of the two inputs ports and provides the result at the output. On the contrary a power splitter shift the phase between the two ports $180^{\circ}$, and effectively provides the difference at the output port. By adding a power combiner to the LISN receiver ports, the CM component will be passed on, while the DM component is suppressed (in the ideal case with infinity damping). A power splitter on the other hand will let the DM component through, while the $\mathrm{CM}$ component is suppressed. Addition and subtraction of the conducted emission measurement can thus be used to form a noise separator.

\section{THEORY}

When designing a noise separator, the question naturally arises as to which theoretical framework and evaluation method, is the most suitable one. The subject has been covered in [2], concluding that S-parameters will present a very suitable framework. This is among others, due to the full characterization obtained of the separator, as compared to the only partial one, which is obtained by using just the transmission coefficients. Accordingly, S-parameters will be the choice of this paper. For a three port device as shown in figure 4, [2] defines:

$$
Z_{I n 1} \approx Z_{0} \frac{1+S_{11}}{1-S_{11}}
$$

and

$$
Z_{\text {In } 2} \approx Z_{0} \frac{1+S_{22}}{1-S_{22}}
$$

$Z_{I n 1}$ and $Z_{I n 2}$ are the input impedances, when the device is terminated by the load $Z_{0}$.

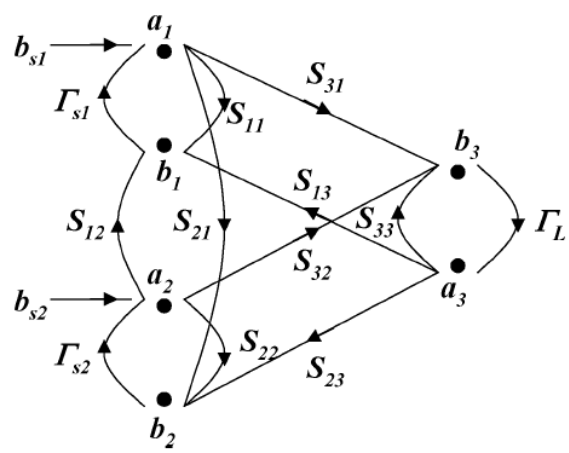

Figure 4: S-parameters of three port device [2].

The rejection ratio $^{1}$ of a differential mode separator are defined as:

$$
\begin{aligned}
D M R R & =\frac{S_{31}}{1+S_{11}+\frac{S_{22} \Gamma_{s 2} S_{12}}{1-S_{22} \Gamma_{s 2}}}-\frac{S_{32}}{1+S_{22}+\frac{S_{21} \Gamma_{s 1} S_{12}}{1-S_{11} \Gamma_{s 1}}} \\
& \approx \frac{S_{31}}{1+S_{11}}-\frac{S_{32}}{1+S_{22}} \\
C M R R & =\frac{S_{31}}{1+S_{11}+\frac{S_{21} \Gamma_{s 2} S_{12}}{1-S_{22} \Gamma_{s 2}}}+\frac{S_{32}}{1+S_{22}+\frac{S_{21} \Gamma_{51} S_{12}}{1-S_{11} \Gamma_{s 1}}} \\
& \approx \frac{S_{31}}{1+S_{11}}+\frac{S_{32}}{1+S_{22}}
\end{aligned}
$$

For a common mode separator the rejection ratios are found from:

$$
\begin{aligned}
D M R R & =\frac{S_{31}}{1+S_{11}+\frac{S_{21} \Gamma_{s 2} S_{12}}{1-S_{22} \Gamma_{s 2}}}-\frac{S_{32}}{1+S_{22}+\frac{S_{21} \Gamma_{s 1} S_{12}}{1-S_{11} \Gamma_{s 1}}} \\
& \approx \frac{S_{31}}{1+S_{11}}-\frac{S_{32}}{1+S_{22}} \\
C M R R & =\frac{S_{31}}{1+S_{11}+\frac{S_{21} \Gamma_{s 2} S_{12}}{1-S_{22} \Gamma_{s 2}}}-\frac{S_{32}}{1+S_{22}+\frac{S_{21} \Gamma_{s 1} S_{12}}{1-S_{11} \Gamma_{s 1}}} \\
& \approx \frac{S_{31}}{1+S_{11}}+\frac{S_{32}}{1+S_{22}}
\end{aligned}
$$

${ }^{1}$ Differential Mode Rejection Ratio (DMRR), Common Mode Rejection Ratio (CMRR). 
The simplified equations 1, 2, 4, 6, 8 and 10 assumes, that the reflection can be neglected. This is normally a valid assumption [2]. The equations of this section will be used in the design, analyze and verification process. Whenever reference is made to any of these equations, the impedance or rejection ratio is obtained by measuring the $\mathrm{S}$-parameters and preforming the relevant calculation.

\section{ANALYSIS}

This section will analyze three commercial power combiners/splitters. The purpose is to establish, which of the devices are suitable for designing a complete noise separator. All three devices are from Mini-Circuits: ZSCJ-2-2, ZFSCJ-2-1 and ZFSC-2-6. The impedance (magnitude and phase) of the devices are shown in figure 5, 6 and 7 . An HP4195A Network/Spectrum Analyzer with associated Transmission/Reflection test set are used to obtain the $\mathrm{S}$-parameters, and the impedances are then calculated based on equations 1 and 2 . The devices were terminated by a $50 \Omega$ resistive load during the measurements.

ZSCJ-2-2 and ZFSCJ-2-1 are both power splitters, while ZFSC-2-6 is a power combiner. [5] suggest to use ZFSCJ-2-1 and ZFSC-2-6 to form a noise separator. However looking at figure 5 and 7, it is clear, that ZSCJ-2-2 is a fare better choice for the splitter than ZFSCJ-2-1. ZSCJ-2-2 and ZFSC-2-6 reflects the $50 \Omega$ resistive load with very little change in phase and magnitude over the targeted frequency range $(150 \mathrm{kHz}-$ $30 \mathrm{MHz}$ ).

Full S-parameter characterization of each device has been preformed. It can be shown, that the presented impedance plots are obtained using equations 1 and 2 . However, due to the limited number of allowed pages for this conference, the individual S-parameters will not be presented.

\section{DESIGN}

It is shown in section III, that ZSCJ-2-2 and ZFSC-2-6 is the suitable choice for designing a complete noise separator. It is suggested to coupled ZSCJ-2-2 and ZFSC-2-6 in parallel allowing for $\mathrm{CM}$ and $\mathrm{DM}$ to be measured simultaneous. In order to fulfill the impedance requirements of the LISN a $25 \Omega$ resistance must be placed in series with the parallel connected splitters/combiners. An schematic of the complete separator and its interface with the LISN are shown in figure 8 , while a photo of the test bench is provided in figure 9.

\section{MEASUREMEnTS}

This section verifies the proposed noise separator through measurements, and provides an example on how to used the separator in a real life measuring setup.

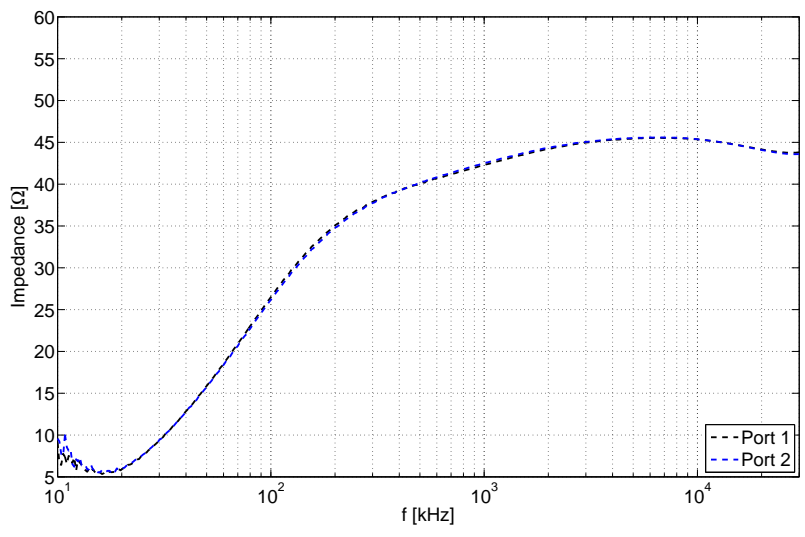

(a) Magnitude.

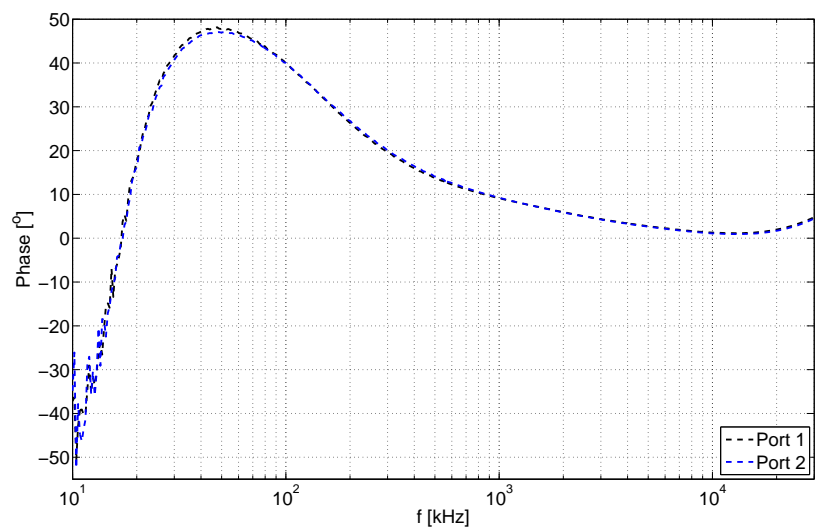

(b) Phase.

Figure 5: Impedance of ZFSCJ-2-1for each of the two input ports.

\section{A. S-parameters}

S-parameters and the equations of 4, 6, 8 and 10 are used to find DMRR and CMRR of the proposed noise separator. The resulting rejection ratios are shown in figure 10. From figure 10 it can be concluded, that the proposed noise separator guarantees a DMRR of $-70 \mathrm{~dB}$ and a CMRR of $-60 \mathrm{~dB}$ over the frequency range of $30 \mathrm{kHz}-30 \mathrm{MHz}$. Ideally the separators should provide infinity damping of either CM or DM, while letting the other one through without any damping. However, in order to establish wether CM or DM are the dominate one, a relative comparison with respect to the total noise spectrum is sufficient. The component which is most compressed compared to the total noise spectrum, will be the dominant one, and thus the one, which the designer of the input filter should pay close attention to. 


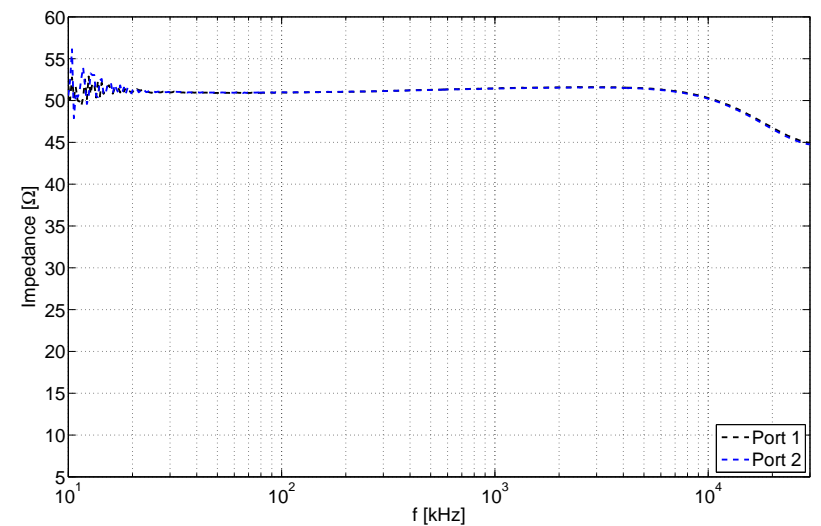

(a) Magnitude.

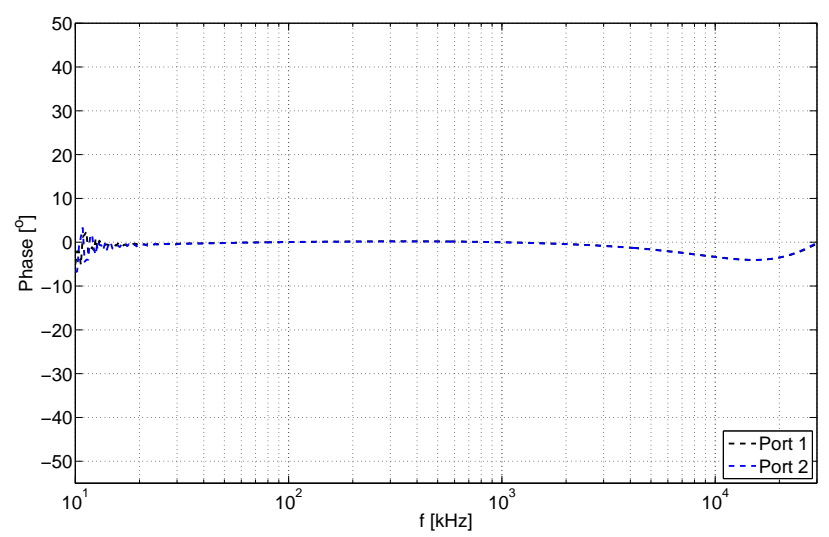

(b) Phase.

Figure 6: Impedance of ZFSC-2-6 for each of the two input ports.

\section{B. LISN impedance}

It is verified that the combined LISN and noise separator does not violate the impedance requirements. The impedance measurement is performed using an AP300 Frequency Response Analyzer (by Ridley Engineering). Figure 11 show the measured result together with the envelop of the impedance tolerance. Clearly the impedance is not violated with the exceptions of the very high frequency range of 10-30 $\mathrm{MHz}$. Extra attention to the layout of the noise separator and its symmetry should be paid in order to counteract this. This is however beyond the scope of the paper.

\section{Spectrums}

In order to illustrate how to use the complete separator setup, spectrum measurements are conducted on a commercial switch mode power supply with is input filter removed. The measurements are conducted with a Rohde \& Schwarz EMI Test Receiver $(20 \mathrm{kHz}-7 \mathrm{GHz})$, and the separator setup

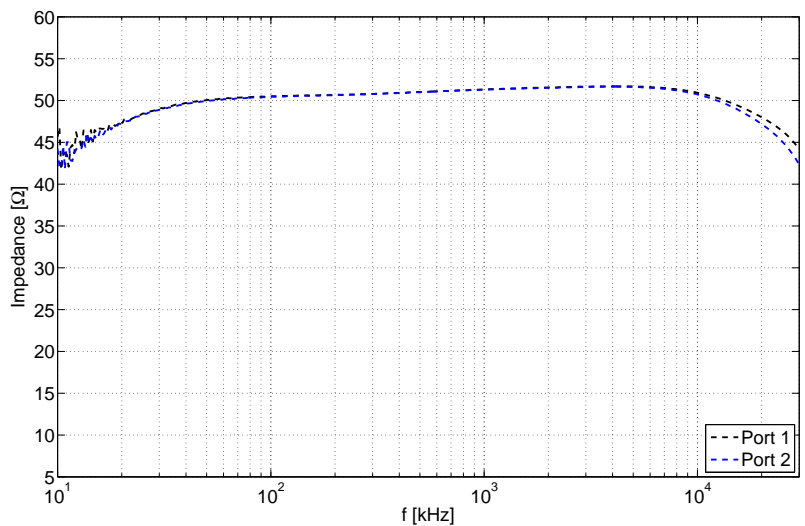

(a) Magnitude.

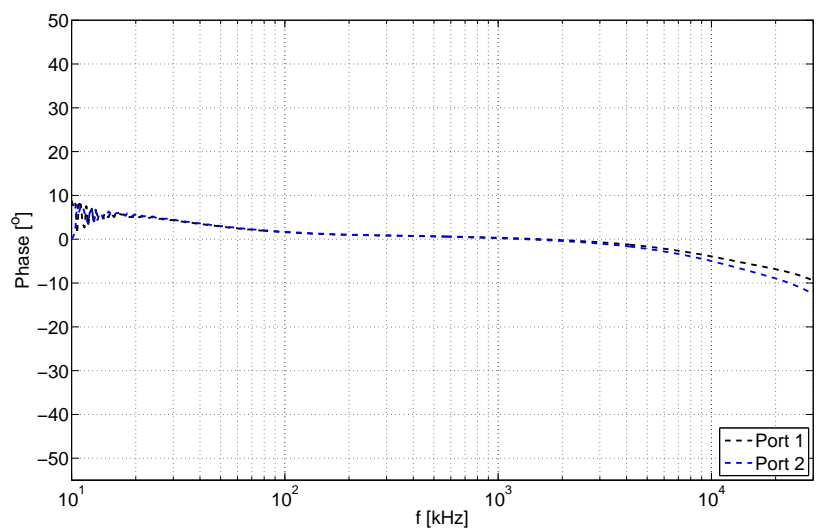

(b) Phase

Figure 7: Impedance of ZSCJ-2-2 for each of the two input ports.

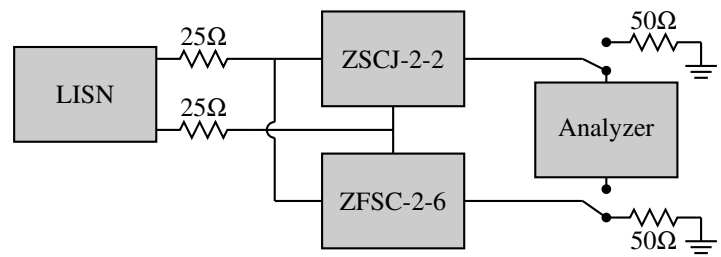

Figure 8: Schematic of complete noise separator with LISN.

described in figure 8 .

The resulting spectrums are shown in figure 12. Limits of conducted emission according to EN55022 are imposed on the plots. Figure 12(a) and 12(b) gives the line and neutral, respectively, with both outlets for the spectrum analyzer terminated by $50 \Omega$. The close similarity of the two plots confirms, that the noise separator does not violates the symmetry of the LISN. 




Figure 9: Complete separator with LISN.

Figure 12(c) and 12(d) shows the output of the ZSCJ22 and ZFSC26, respectively. CM is the component suppressed in figure 12(c), while DM is suppressed in figure 12(d).

Traditionally the designer of a EMI filter will look at figure 12(a) and/or 12(b). He will then assume, that DM emission are associated with low frequency switching, while CM emission are due to the higher frequency switching components [9]. This leads to the conclusion, that the DM part of the input filter should provide about $45 \mathrm{~dB}$ of damping, while the $\mathrm{CM}$ part must provide roughly $30 \mathrm{~dB}$ damping. However if the designer used the proposed separator, he will arrive to a bit different conclusion. First most, it should be stressed, that the spectrums of 12(c) and 12(d) must be viewed with reference to 12(a) and 12(b), as one essential wishes to compared the total noise with the suppressed noise. Figure 12(c) shows that the spectrum is damped over the entire frequency range, with the high frequencies being the most suppressed ones. From figure $12(\mathrm{~d})$ one sees, that the spectrum only is suppressed in the very low frequency range (below 400 $\mathrm{kHz}$ ). It can thus be concluded, that $\mathrm{CM}$ emission is the dominant one in the considered case. The CM part of the input filter should thus be emphasized during the design phase.



(a) ZSCJ22.

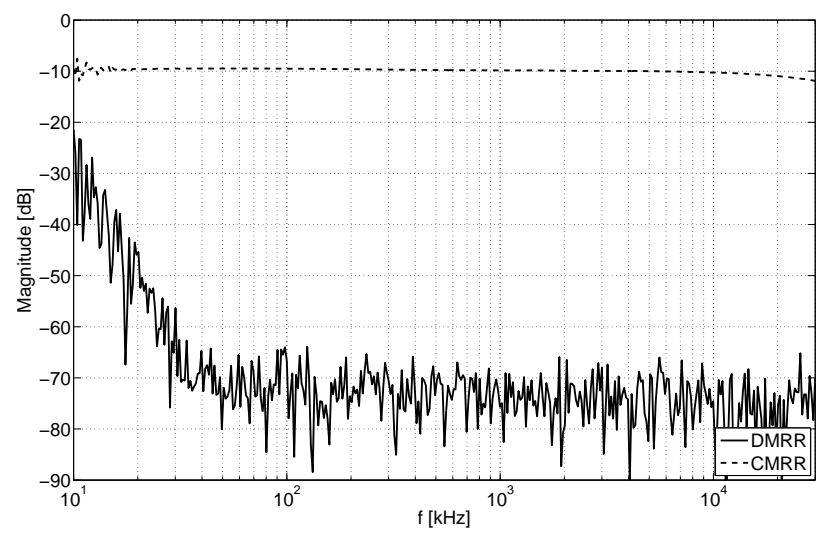

(b) ZFSC26.

Figure 10: Rejection ratios of the proposed noise separator.

\section{CONCLUSION}

This paper presents a conducted emission noise separator based on commercial power combiners/splitters. Characterization are performed using S-parameters, and based on these results a design for the noise separator is proposed. The noise separator is verified through experimental measurements. An example is presented, where the separator is used for measuring common and differential mode noise of a commercial switch mode power supply with its input filter removed.

\section{REFERENCES}

[1] J. Stahl, D. Kuebrich, and T. Duerbaum, "Characterisation of an effective emi noise separation including a standard lisn," in Proc. URSI Int Electromagnetic Theory (EMTS) Symp, 2010, pp. 13-16.

[2] S. Wang, F. C. Lee, and W. G. Odendaal, "Characterization, evaluation, and design of noise separator for conducted emi noise diagnosis," vol. 20, no. 4, pp. 974-982, 2005.

[3] M. C. Caponet and F. Profumo, "Devices for the separation of the common and differential mode noise: design and realization," in Proc. Seventeenth Annual IEEE Applied Power Electronics Conf. and Exposition APEC 2002, vol. 1, 2002, pp. 100-105. 


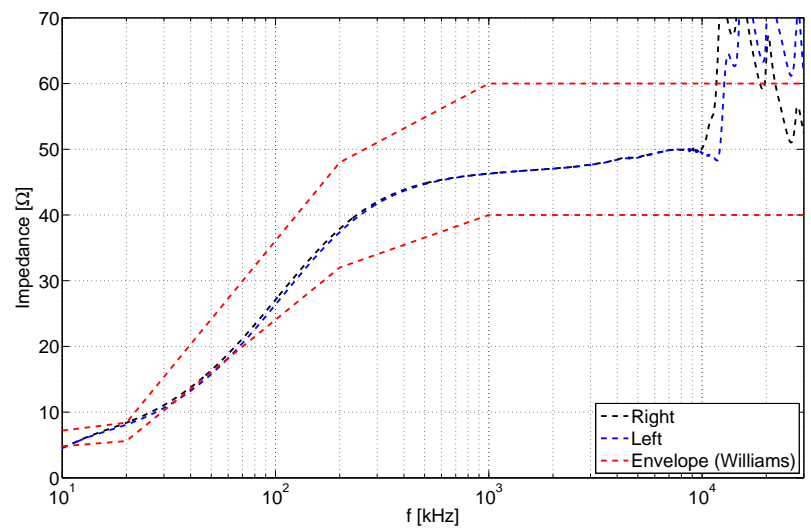

Figure 11: Input impedance of LISN.

[4] M. C. Caponet, F. Profumo, L. Ferraris, A. Bertoz, and D. Marzella, "Common and differential mode noise separation: comparison of two different approaches," in Proc. PESC Power Electronics Specialists Conf. 2001 IEEE 32nd Annual, vol. 3, 2001, pp. 1383-1388.

[5] T. Guo, D. Y. Chen, and F. C. Lee, "Separation of the common-modeand differential-mode-conducted emi noise," vol. 11, no. 3, pp. 480-488, 1996.

[6] A. A. Ramesh, B. Subbarao, and R. Sivaramakrishnan, "Design of low cost common and differential mode noise diagnostic circuit," in Proc. 9th Int ElectroMagnetic Interference and Compatibility (INCEMIC) Conf, 2006, pp. 343-348.

[7] H.-L. Su and K.-H. Lin, "Computer-aided design of power line filters with a low cost common and differential-mode noise diagnostic circuit," in Proc. EMC Electromagnetic Compatibility 2001 IEEE Int. Symp, vol. 1, 2001, pp. 511-516.

[8] M. J. Nave, "A novel differential mode rejection network for conducted emissions diagnostics," in Proc. IEEE 1989 National Symp. Electromagnetic Compatibility, 1989, pp. 223-227.

[9] T. Williams, EMC for Product Designers, Fourth, Ed. Elsevier Ltd, 2007.

[10] Y.-S. Lee and Y. Shu, "Line filter design of switching mode power supply using software approximation for conducted emission separation," in Proc. Fifth Int. Conf. Power Electronics and Drive Systems PEDS 2003, vol. 2, 2003, pp. 1339-1344.

[11] J. Stahl, D. Kuebrich, and T. Duerbaum, "Modification and characterization of a standard lisn for effective emi noise separation," in Proc. Int Electromagnetics in Advanced Applications (ICEAA) Conf, 2010, pp. 39-42.

[12] C. R. Paul and K. B. Hardin, "Diagnosis and reduction of conducted noise emissions," vol. 30, no. 4, pp. 553-560, 1988.

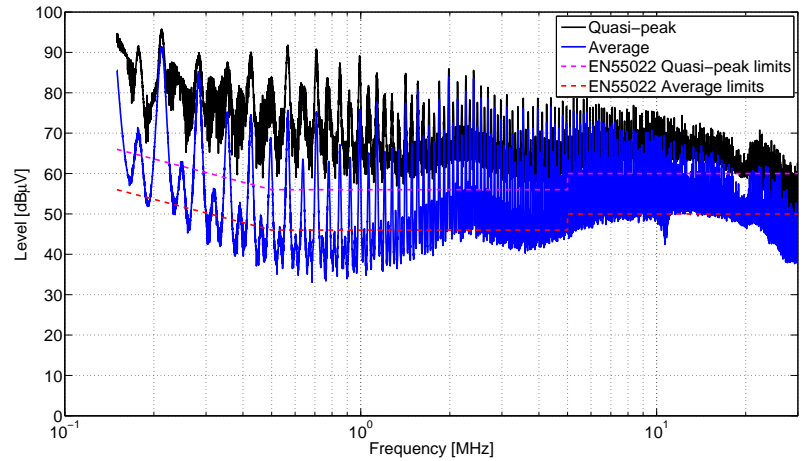

(a) Neutral.

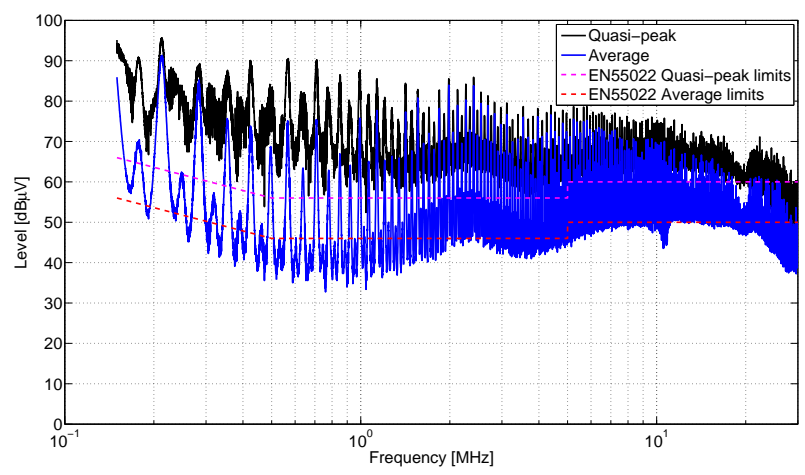

(b) Line.

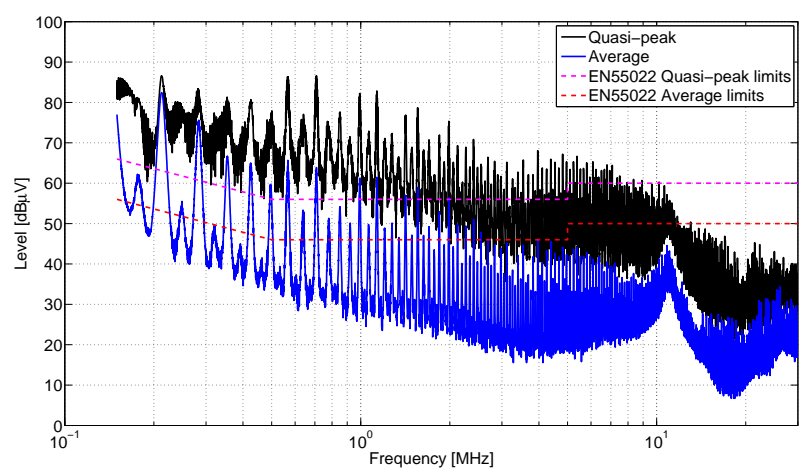

(c) ZSCJ22.

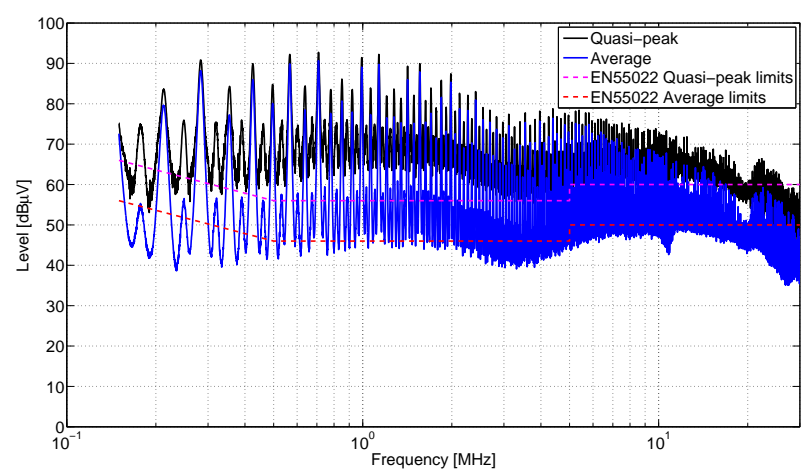

(d) ZFSC26.

Figure 12: Spectrum measurements. 